\title{
Engaging countries and strengthening partnerships towards better maternal and child health
}

Citation: Engaging countries and strengthening partnerships towards better maternal and child health. East Mediterr Health J. 2018;24(12):1181-1183 https://doi.org/10.26719/2018.24.12.1181

Copyright @ World Health Organization (WHO) 2018. Some rights reserved. This work is available under the CC BY-NC-SA 3.0 IGO license (https:// creativecommons.org/licenses/by-nc-sa/3.o/igo).

\section{Introduction}

Inadequate progress in reducing maternal, neonatal and child mortality had been made in the Eastern Mediterranean Region (EMR) (1). WHO had been founded on the principle that all people should be able to realize their right to the highest possible level of health. In line with this, and the regional Vision 2023 for the health and wellbeing of people in the Region and Thirteenth General Programme of Work (GPW 13) (2), WHO had developed and updated guidelines and tools to help strengthen national reproductive and maternal health services covering preconception care, family planning, antenatal and intrapartum care, medical eligibility criteria and selected practice recommendations for contraception use, and Robson classification (3) and non-clinical interventions to reduce unnecessary caesarean sections (4).

In support of these guidelines, the World Health Organization Regional Office for the Eastern Mediterranean (WHO/EMRO) held a regional meeting for engaging countries and strengthening partnerships towards better maternal and child health in Amman, Jordan, from 21 to 23 November 2018. The objectives of the meeting were to:

- build the technical capacity of reproductive, maternal, neonatal, child and adolescent health focal points at WHO country offices for adopting the updated maternal and child health guidelines and recommendations (5), and identify key priorities in line with the Sustainable Development Goals (SDGs) and WHO's Thirteenth General Programme of Work (GPW 13);

- discuss tools and approaches to translate the maternal and child health shared guidelines into action/ practice to reach SDG/GPW 13 targets;

- strengthen the skills of WHO focal points to mobilize domestic and international resources through innovative approaches and ensure sustainable financing mechanisms for maternal and child health programmes; and

- update participants on target setting and monitoring of national reproductive and maternal health indicators using linkages between the SDGs and GPW 13 targets.
The meeting was attended by 88 participants from most Member States of the Region, as well as experts from Jordan, Morocco, Tunisia, and United Kingdom, and staff from the Amman Jordanian Association, Marie Stoops International (Jordan), UNAIDS, United Nations Relief and Works Agency for Palestine Refugees (UNRWA), and WHO country, regional, and headquarter offices.

\section{Summary of discussions}

\section{Preconception care}

A limited number of countries have adopted a comprehensive approach to deliver preconception care services, with most countries instead providing premarital services targeting couples prior to marriage. National preconception care programmes need to be established and/or strengthened in line with the key 10 steps adopted by the $65^{\text {th }}$ session of the Regional Committee held in Khartoum, Sudan, in October $2018(-6)$.

At the country level, there are is some political commitment to preconception care, policies and regulations, and some national strategies include preconception care. Across the Region, preconception care is being implemented either with some key interventions or with full coverage (including vaccination and nutrition interventions). Some countries operate very strong premarital health programmes that facilitate the implementation of preconception care.

At the health facility level, implementation of preconception care is limited by the lack of: institutionalization and integration; contextualized preconception care guidelines; health-seeking behaviour; and involvement of the private sector.

At the community level, health providers have limited ability to ask culturally-sensitive questions regarding family history of congenital anomalies, mental illness, contraceptive use, sexual activity, smoking and alcohol consumption, and domestic violence.

\section{Strengthening family planning services and policies}

There was discussion on the WHO updated family planning tools and recommendations regarding medical eligibility criteria for contraception, safe contraception use, managing the side effects of modern family planning

\footnotetext{
This report is extracted from the Summary report on the Regional meeting for engaging countries and strengthening partnerships towards better maternal and child health, Amman, Jordan, 21-23 November 2018 (http://applications.emro.who.int/docs/IC_Meet_Rep_2018_EN_20789.pdf?ua=1).
} 
methods, and eligibility criteria for the postpartum period and for women living with HIV undergoing antiretroviral therapy.

Integrating $\mathrm{WHO}$ guidance into national guidelines involves four stages: an exploration phase, including mapping current country family planning guidelines and status of use; an installation phase to launch practical preparations for a revised family planning programme; an initial implementation phase, including a roll out of the revised family guidelines; and a full implementation stage of integrating the guidance on medical eligibility criteria and selected practice recommendations into national guidelines.

\section{WHO recommendations on antenatal care for a positive pregnancy experience}

Antenatal care remains a key opportunity for timely and appropriate evidence-based actions for health promotion, disease prevention, screening and treatment. Challenges in providing quality pregnancy care at health facilities include inequitable access to antenatal care services, limited financial resources to ensure better coverage and quality, the limited skills of healthcare providers, and the use of out-dated guidelines and recommendations.

Adopting the 2016 WHO guidelines on antenatal care for a positive pregnancy experience (7) will improve the quality of pregnancy care at health facilities. This involves a transition from the four antenatal visits of the focused antenatal care model to eight contacts as specified in the updated WHO recommendation, with prioritization of person-centered health and well-being through provision of respectful care that takes into account women's views and the optimization of service delivery within health systems.

\section{WHO recommendations on intrapartum care for a positive childbirth experience}

The WHO recommendations on intrapartum care (8) address the increasing use of labour interventions in the absence of clear indications by identifying the most common practices used throughout labour and establishing standards of good practice for the conduct of uncomplicated labour and childbirth. The concept of experience of care is critical in ensuring high-quality labour and childbirth care and improved woman- centered outcomes, and is not just complementary to routine clinical practices. There is a need to adopt the 2018 WHO intrapartum care recommendations to improve the coverage and quality of care services. The recommendations should inform the development of relevant national- and local-level health policies and clinical protocols.

\section{Robson classification and WHO guidelines on non-clinical interventions to reduce unnecessary caesarean section}

The average rate of caesarean section in the Region is $21 \%$ and ranges from one of the highest country rates in the world (52\% in Egypt) to the lowest ( $2 \%$ in Somalia) (4). The factors behind the high rates of caesarean section include the lack of national policy/guidelines regarding child birth practice, a profit-motivated private sector, improper management of normal labour, irrational use of obstetric interventions, lack of communication between health care professionals and women, and reduced midwifery input in the childbirth process (4). There is a need to adopt the 2018 WHO recommendations on non-clinical interventions to reduce unnecessary caesarean sections through interventions targeting women, communities, health care professionals and the health system.

\section{Next steps for Member States}

- Proceed with the necessary actions to implement the WHO recommended interventions and programme steps for strengthening preconception care in Member States (during 2019).

- Expand capacity-building activities for family planning in the public and private sectors using WHO updated family planning tools and recommendations (during 2019).

- Adopt and provide training in and implement WHO recommendations on antenatal and intrapartum care (during 2019).

- Use Robson classification to monitor the quality of childbirth care and reduce unnecessary caesarean sections (during 2019).

- Conduct formative research on non-clinical interventions for reducing unnecessary cesarean sections and build on the findings of these research activities (during the period: 2019-2020).

\section{References}

1. World Health Organization. Maternal, newborn, child and adolescent health. Geneva: World Health Organization; 2018 (https:// www.who.int/maternal_child_adolescent/en/).

2. The Seventy-first World Health Assembly. Thirteenth general programme of work 2019-2023. Geneva: World Health Assembly; 2018 (https://www.who.int/about/what-we-do/gpw-thirteen-consultation/en/).

3. World Health Organization. Robson classification: implementation manual. Geneva: World Health Organization; 2017 (https:// www.who.int/reproductivehealth/publications/maternal_perinatal_health/robson-classification/en/). 
4. World Health Organization. WHO recommendations: Non-clinical interventions to reduce unnecessary caesarian sections. Geneva: World Health Organization; 2018 (http://apps.who.int/iris/bitstream/handle/10665/275377/9789241550338-eng.pdf?ua=1).

5. World Health Organization. Standards for improving quality of maternal and newborn care in health facilities. Geneva: World Health Organization; 2016 (https://www.who.int/maternal_child_adolescent/documents/improving-maternal-newborn-care-quality/en/).

6. World Health Organization Regional Office for the Eastern Mediterranean (WHO/EMRO). 65th Session of the WHO Regional Committee for the Eastern Mediterranean. Cairo: WHO/EMRO; 2018 (http://applications.emro.who.int/docs/RC65_2018_Daily_Journal_2_20627_EN.pdf?ua=1).

7. World Health Organization. WHO recommendations on antenatal care for a positive pregnancy experience. Geneva: World Health Organization; 2016 (http://apps.who.int/iris/bitstream/handle/10665/250796/9789241549912-eng.pdf;jsessionid=CF45FCD6A3FA28D35333E1C264B81283?sequence=1).

8. World Health Organization. WHO recommendations: Intrapartum care for a positive childbirth experience. Geneva: World Health Organization; 2018 (http://apps.who.int/iris/bitstream/handle/10665/260178/9789241550215-eng.pdf?sequence=1). 